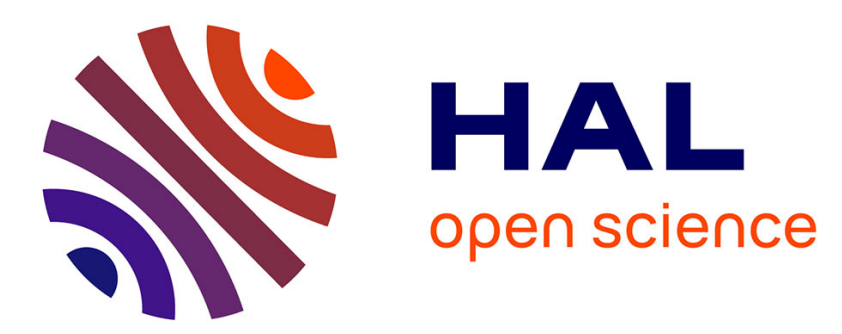

\title{
Low Energy Electrons Emitted in Collisions of Heavy Ions with Solid State Targets
}

\author{
N. Lineva, C. Kozhuharov, S. Hagmann, M. Krämer, G. Kraft
}

\section{To cite this version:}

N. Lineva, C. Kozhuharov, S. Hagmann, M. Krämer, G. Kraft. Low Energy Electrons Emitted in Collisions of Heavy Ions with Solid State Targets. The Seventh International Symposium on Swift Heavy Ions in Matter, Jun 2008, Lyon, France. hal-00256639

\section{HAL Id: hal-00256639 \\ https://hal.science/hal-00256639}

Submitted on 25 Aug 2008

HAL is a multi-disciplinary open access archive for the deposit and dissemination of scientific research documents, whether they are published or not. The documents may come from teaching and research institutions in France or abroad, or from public or private research centers.
L'archive ouverte pluridisciplinaire HAL, est destinée au dépôt et à la diffusion de documents scientifiques de niveau recherche, publiés ou non, émanant des établissements d'enseignement et de recherche français ou étrangers, des laboratoires publics ou privés. 


\section{Low Energy Electrons Emitted in Collisions of Heavy lons with}

Solid State Targets

Natalia Lineva ${ }^{1, *}$, C. Kozhuharov ${ }^{1}$, S. Hagmann ${ }^{1,2}$, M. Krämer ${ }^{1}$, G. Kraft ${ }^{1}$

${ }^{1}$ GSI, Planckstr. 1, 64291 Darmstadt, Germany

${ }^{2}$ Goethe Universität Frankfurt, IKF, Max-von-Laue Weg 1, 60438 Frankfurt am Main, Germany

${ }^{*}$ Corresponding author. Tel. +49 615171 1387: fax: +49 615971 2044;

E-mail addresses: $\underline{n . l i n e v a @ g s i . d e, ~ c . k o z h u h a r o v @ g s i . d e ~}$ 


\section{Abstract}

Detailed knowledge of low-energy electron emission in collisions of swift ions with solids and gases is of utmost importance for the modeling of the dose distributions and track structures at small distances $(<20 \mathrm{~nm})$ in mixed radiation fields, particularly for the Local Effect Model, LEM, developed and used at GSI for the prediction of ion radiation effects in ion radiotherapy and related fields. At GSI, we have launched a project aimed at systematic investigations of the energy and angular distributions of low energy (sub-keV) electrons emitted from solids. We have commissioned an electrostatic toroidal spectrometer at the UNILAC accelerator, and we have developed a method to identify, study and analyze problems caused by the condition and constitution of the target foils and their surfaces. For this, we combined measurements of electrons emitted in collisions of low-energy electron beams $(500 \mathrm{eV}, 1 \mathrm{keV})$ with results of dedicated Monte Carlo simulations. We utilized the findings of these studies for the investigation of electrons emitted from the same targets in ion-atom collisions for the data analysis, and we report on results of our first measurements with carbon projectiles.

PACS: 34.50.-s; 34.50.Fa; 34.80.-b; 87.50.cm; 87.64.ks

Keywords: Electron emission; Electron spectrometer, Low-energy electrons, Swift ions 


\section{Introduction}

The knowledge of the biological actions of heavy ions is of fundamental interest both for radiation protection-particularly the protection of astronauts against cosmic rays and proton wind-as well as for the application of particle beams in medicine like the heavy-ion tumor therapy developed in the last twenty years by the Biophysics Division of GSI Darmstadt. A recent comprehensive review on the ion radiotherapy at GSI can be found in Ref. [1]. The interaction of swift charged particles penetrating matter differs from the interaction of low-LET radiation like $x$ and $\gamma$-rays on various levels: The energy loss of the projectile as well as the energy deposited in matter scales with the second power of the effective charge, increasing, thus, the importance of heavier ions like carbon and iron as compared to protons. The strong velocity dependence of the projectile energy loss gives rise to an inverse profile of the 'macroscopic' dose. The dose deposition is relatively low at high velocities, i.e. at the entrance to the irradiated volume, and increases moderately with increasing penetration depth. It culminates in the stopping region in the typical Bragg maximum with an unparalleled peak-to-entrance dose ratio.

For ion radiotherapy as well as for radioprotection purposes, however, another significant difference exists on the microscopic level. Swift ions dissipate their energy in numerous ion-electron collisions, with very small energy transfers and practically no effect on the ion trajectory, i.e. the angular straggling of the ions is small. For projectile energies higher than $100 \mathrm{keV} / \mathrm{u}$, approximately (2/3) of the energy is converted into target ionization. The emitted electrons have low energy, which they dissipate in further collisions, creating a cascade of collisions with target electrons. Thus, to- 
gether with the primary ionization stemming from the ionic projectile, a track of high ionization density is created. The local dose within such a track increases steeply with decreasing distance to the primary ion path and can reach values exceeding the average macroscopic dose by orders of magnitude. It is this high local dose deposition, which gives rise to the increased biological effectiveness of ion radiation, apart from their absorbed dose deposition. The associated radiation effects can be calculated or at least estimated with good accuracy, using models such as LEM [1,2]. A prerequisite, however, is the precise knowledge of the inner part of the track (below $20 \mathrm{~nm}$ ), which in turn requires the knowledge of the energy (and angular) distribution of the low-energy electrons released during the passage of the ions. Since electrons are not only released by the primary ion, but also as a result of secondary ionization, the emitted electrons are both messenger of as well as originator for the ionization, and the knowledge of their energy and spatial distributions is essential, since the ionization of tissue molecules—with the resulting dissociation and/or with the buildup of free radicals - is the base for the understanding of the biological effects of radiation, both its action as well as the radiation damage. The dimensions of interest for the biological effects range from a few micrometers (i.e. the dimensions of cell nuclei) down to a few nanometers (two DNA strands separated by this distance are the most sensitive structure). It is, thus, mandatory to understand the spatial (radial) distribution of the locally deposited dose even at very small distances, i.e. it is important to know the energy distributions of emitted electrons down to very low energies, their angular distributions, the angular straggling, as well as the mechanisms that govern the slowing down of electrons with initially higher energies. 
The radial dose distributions with fluid and/or solid-state targets cannot be directly measured for distances as small as a few $\mathrm{nm}$. The available data originate from experiments that have been performed in much larger volumes, in dedicated gas counters, filled with tissue-equivalent gases at low pressure. One assumes that the observed effects scale with the density ratio of the gas and the tissue (cf. [3] for a review). The experimental data exhibit a radial fall-off of the locally deposited dose inversely proportional to the second power of the distance from the track center. The $\left(1 / \mathrm{r}^{2}\right)$ radial dose distribution has been corroborated by Monte Carlo simulations [4-6] and by model calculations $[1,7]$. Prerequisites for the Monte Carlo simulations are the doubly differential cross sections for electron emission with respect to the electron energy and emission angle. Monte Carlo simulations are needed for all models used for ion-radiotherapy planning: both for the Local Effect Mode, LEM [1,2]; as well for the microdosimetry [8]

It is, however, easy to imagine, that differences between gas and solids have to exist. In gases under single collision conditions, for instance, loosely bound electrons can be ionized even at very large impact parameters with very large geometrical cross sections, since the required momentum and energy transfers are tiny. Such impact parameters are much larger than the interatomic/intermolecular distances or the lattice constant of solid-state targets. In other words, due to the much higher density, target atoms at large impact parameters are screened by the target atoms at smaller impact parameters. Furthermore, the ions passing through the solids create a Mach cone-like wake with a time dependent potential, superimposed on the track potential caused by the very high ionization density. Due to inherent difficulties in the detection of low-energy electrons as well as in disentangling the results of multiple collisions 
and of the superimposed transport mechanisms, the (not so numerous) existing experiments with solid-state targets are usually tailored to study some specific features, as for instance the electron temperature inside the heavy-ion track. For a recent review—it also includes a comprehensive list of the open questions—confer Ref. [9]. 


\section{Experiment}

We have initiated a program to systematically investigate the low energy electron emission from solids. For this, we have customized the X3-beamline at the UNILAC accelerator, and we have installed an electrostatic electron spectrometer there, which was developed by one of us, Siegbert Hagmann. Figure 1 shows the spectrometer. The well-collimated ion beam enters from the left ('beam entrance', lower left corner of the picture). The collimators kept the beam spot smaller than $0.5 \mathrm{~mm}$. The target is in the middle of the shielded target chamber, mounted under $110^{\circ}$ with respect to the beam axis. Up to eight targets can be mounted on a target wheel and brought into position without venting the vacuum chamber. The spectrometer is rotationally symmetric with respect to the axis that crosses the beam axis perpendicularly at the target position. Electrons, emitted under all polar angles are energy analyzed and focused onto an MCP (micro channel plate) detector with a position-sensitive delay-line read-out. The focus is ring-shaped with a diameter of $2 \mathrm{~cm}$ on an average. The detector (not shown) is located on the top of the figure. We have performed simulations of the electron trajectories with the SIMION [10] software. The relative energy resolution and the acceptance in azimuth are defined by the analyzing slits and amount to $\Delta \mathrm{E} / \mathrm{E}=2 \%$ and to $2.8^{\circ}$, respectively. The simulated polar angle resolution of the spectrometer amounts to $0.3^{\circ}$, the position resolution of the detector reduces this number to $1.7^{\circ}$, nonlinearities and distortions of the delay-line readout led to further degradation of the polar angle resolution. The electron spectra were measured by scanning repeatedly up and down the spectrometer fields in 2048 equidistant steps for electron energies from 0 to $1 \mathrm{keV}$. The integrated Faraday Cup current per energy 
step provided the normalization of the spectra. Special care was taken to minimize the dead time and to correct it properly.

The targets were standard thin foils produced in the GSI target laboratory and consisted of 100 atomic layers on an average: C $\left(4.7 \mu \mathrm{g} / \mathrm{cm}^{2}\right), \mathrm{Ni}\left(45 \mu \mathrm{g} / \mathrm{cm}^{2}\right), \mathrm{Ag}(38$ $\left.\mu \mathrm{g} / \mathrm{cm}^{2}\right)$, and $\mathrm{Au}\left(40 \mu \mathrm{g} / \mathrm{cm}^{2}\right)$. The target frames had an opening of $5 \mathrm{~mm}$ in diameter. The projectiles were protons, $\mathrm{H}_{3}{ }^{+}$, and $\mathrm{C}^{2+}$. Additionally, we used monoenergetic 500 $\mathrm{eV}$ and $1 \mathrm{keV}$ electron beams from an electron gun to bombard the targets. 


\section{Results and Discussion}

Since low energy electrons traversing the target do not produce a track with an ionization density as high as those produced by ions, we started our investigations by analyzing the energy and angular distribution of electrons emitted after impact of 1 $\mathrm{keV}$ electrons on carbon targets with a nominal thickness of $4.7 \mu \mathrm{g} / \mathrm{cm}^{2}$ and 11 $\mu \mathrm{g} / \mathrm{cm}^{2}$, respectively. It should be emphasized, that even for the thinnest target the single collision condition is not fulfilled. The multitude of interlaced and interdependent scattering processes produces a picture, which is not easy to be directly interpreted. We combined therefore our analysis with dedicated Monte Carlo simulations with the TRAX code [11] written by one of us, Michael Krämer, which treats each basic interaction of an electron or a heavy ion separately and does not require models for multiple scattering, angular straggling etc. as an input. The basic processes taken into account for electrons are: elastic scattering, excitation, and ionization. This bottom-up approach in simulating the emission and transport phenomena of electrons makes the TRAX code very well-suited for our purposes.

We started our investigations with measurements of $1 \mathrm{keV}$ electrons from an electron gun scattered on $4.7 \mu \mathrm{g} / \mathrm{cm}^{2}$ and $11 \mu \mathrm{g} / \mathrm{cm}^{2}$ carbon targets, and we performed numerical simulations with TRAX for a variety of target thicknesses: from $1 \mu \mathrm{g} / \mathrm{cm}^{2}$ to 10 $\mu \mathrm{g} / \mathrm{cm}^{2}$. The results-differential electron yields in the energy region up to $1 \mathrm{keV}-$ are displayed in Fig. 2 as a function of the polar angle. For all target thicknessesexcept for $1 \mu \mathrm{g} / \mathrm{cm}^{2}$-the simulated distributions (small symbols) exhibit the typical behavior of the electron diffusion process. The electrons emitted backwards show the 
same angular distribution and the same yields; obviously, the back diffusion thickness for $1 \mathrm{keV}$ electrons in carbon is smaller than $2 \mu \mathrm{g} / \mathrm{cm}^{2}$. The diffusion in the forward hemisphere is characterized by the same shape of the angular distribution; here, however, the transmitted intensity decreases with an increasing target thickness. Compared to the simulation, the measured data for $4.7 \mu \mathrm{g} / \mathrm{cm}^{2}$ (big squares) are consistent with the simulation for $3.6 \mu \mathrm{g} / \mathrm{cm}^{2}$, whereas the measured transmission for $11 \mu \mathrm{g} / \mathrm{cm}^{2}$ (big circles) correspond to a simulated thickness slightly larger than 5 $\mu \mathrm{g} / \mathrm{cm}^{2}$. Since the target thickness, averaged over a larger area, is very close to the nominal one, the finding can be explained by large fluctuations of the thickness. Since there were some visible pinholes, we can assume that the thickness fluctuations are probably as large as the nominal thickness itself.

We compared the simulated to the measured energy distributions of electrons emitted in selected angular ranges and found a very good agreement of the spectral shapes when we assumed that the effective target thicknesses are 3.6 instead of 4.7 $\mu \mathrm{g} / \mathrm{cm}^{2}$ and 5.4 instead of $11 \mu \mathrm{g} / \mathrm{cm}^{2}$. For the nominal thicknesses (and for all other thicknesses listed in Fig. 2) we observed pronounced shifts of the maxima of the energy distributions, as expected for larger (and/or for a different) energy loss of the transmitted electrons. These findings also underline the fact, that-even for solidstate target thicknesses as small as the ones used-the transport phenomena do not scale linearly with the thickness fluctuations and do not simply average over the possible thickness ranges.

Based on these findings we analyzed the spectra of electrons emitted in collisions of 3.6 and $11.4 \mathrm{MeV} / \mathrm{u}$ carbon ions impinging on the same carbon target, the one with the nominal thickness of $4.7 \mu \mathrm{g} / \mathrm{cm}^{2}$. Additionally, we bombarded Ni, Ag, and Au tar- 
gets. In Figure 3, we compare our results for the different targets with common simple conventional theory and with the results of our TRAX simulations. We used the modified Rutherford formula as described in Ref. [12], which according to the authors 'works remarkably well for both soft-collisions and binary-encounter electrons.' We used the tabulated binding energies from Ref. 13 and 14. As usual, we took solely the 'active' electrons (those with velocities smaller than the projectile velocity) into account. We calculated the evolution of projectile charge states within the targets with the Etacha code [15], and we used the numbers to weigh the square of the charge state needed to calculate the cross sections. We used the average charge state after the target to obtain the number of projectiles from the measured Faraday cup current. We would like to emphasize that the effective charge state can vary quite strongly with the fluctuations of the target thickness.

The measured and calculated energy distributions are displayed in the left part of Fig. 3. We see that a large fraction of the produced low-energy electrons is stopped and absorbed in the target. This explains the large discrepancies between the theory and experiment in this energy region. Even more important, we see that even the relative experimental and calculated yields do follow the same trend; the measured and calculated cross sections show different sequences, i.e. a different scaling with the atomic number of the target. Apparently, the electron transport phenomena play an important role. In the right part of the figure we compare the measured data for the carbon target with our TRAX simulations. For the simulations, we did not include the electrons stripped from the projectile. The projectile electrons move predominantly with velocities close to that of the projectile itself and are eventually slowed down in the target. In the investigated sub-keV energy region, we expect to see more such 
electrons at the lower bombarding energy as can be seen in the upper right spectrum in Fig. 3. The lower right part shows that the TRAX simulation for He-like Cprojectiles reproduces the measured data very well. The structures visible in the measured spectra are $\mathrm{C}$ and $\mathrm{O}$-Auger lines, which we did not include in the simulations. Since those lines appear in all measured spectra, they can be attributed to adsorbents from the residual gas of the vacuum chamber. Only a fraction of the carbon Auger electrons from the carbon target is emitted from the target itself (the range of the C-Auger electrons in $\mathrm{C}$ is appr. $1 \mu \mathrm{g} / \mathrm{cm}^{2}$ ). Although the C-Auger electrons from the other targets and the O-Auger electrons are emitted from the target surfaces, the lines exhibit very long low-energy tails. Keeping in mind, that these electrons are emitted isotropically, this observation demonstrates the role of the back diffusion processes. 


\section{Summary}

We have initiated a series of measurements of low-energy electrons emitted in collisions of heavy ions solid-state targets. For this, we have optimized and commissioned an electrostatic electron spectrometer. Prior to analyzing our heavy-ion measurements, we have developed a method to investigate the solid-state targets by combining dedicated Monte Carlo simulation with measurements of electrons emitted by bombardment of the targets with monochromatic electron beams. The method demonstrates that the multitude of the involved electron transport processes cannot be linearly averaged and that the inhomogenities of the target thickness play an important role that can obscure the observation and, thus, complicate and hamper the interpretation. This is also illustrated by the large quantitative and qualitative discrepancies between the measured spectra and those calculated with a simple but wellestablished theory, albeit the latter successfully describes the electron emission from single collisions in gases. On the other hand, within the experimental uncertainties of this pilot project, we observe a good agreement of the measured and TRAXsimulated data, which corroborates the bottom-up approach of TRAX in treating the emission and transport phenomena. since the TRAX simulation is based on data and theories for gaseous targets, we are led to conclude that-within the experimental uncertainties-the measured electron emission pattern is very similar to the one known for gases. We would like to emphasize that-at present—our experimental uncertainties are rather large, and that the conclusion is partially based on the assumption that the TRAX simulations describe properly the processes involved. This assumption is justified by the achievements of TRAX within the framework of the ion radiotherapy at GSI. Nonetheless, it will be interesting to compare the TRAX results 
with the results of similar numerical simulations. Furthermore, the reported first results of our measurements accentuate the need for more systematic investigations, for an increased precision of the measured data as well as for an improved reliability of their interpretation. Based on the present results, we have developed a detailed program for further investigations [16]. To mention one example in particular, we will implement extremely thin $0.5 \mu \mathrm{g} / \mathrm{cm}^{2}$ diamond-like targets. This will allow us to utilize the line shape analysis of the carbon Auger lines as a diagnostic tool pertinent to our studies. Additionally, we will use a single crystal CVD diamond detector both as a target as well as a projectile detector. The use of such detectors will allow us to correlate event-by-event the emitted electrons to the instantaneously deposited dose of a single projectile ion and will constitute a novel dosimetry method.

Acknowledgements: Discussions with Eleni Berdermann, Markus Bender, Peter Forck, Holger Kollmus, Vitaly Liechtenstein, and Herman Rothard are highly appreciated. 


\section{References}

[1] G. Kraft, http://www.gsi.de/forschung/bio/tumorverein eng190607 xs.pdf (2007) last retrieved on 29-May-2008, Verein zur Förderung der Tumortherapie mit schweren lonen e.V. ISBN: 3-9811298-2-2; 978-3-9811298-2-3

[2] M. Scholz, G. Kraft, Radiat. Prot. Dosim. 52 (1994) 29 and M. Scholz, G. Kraft, Rad. Res. 161 (2004) 612; also cf. M. Scholz, G. Kraft, Workshop on Biophysical Modeling on Radiation Effects, Padua, Italy, 1991

[3] H.H. Rossi, M. Zaider, Microdosimetry and its Applications, Springer Verlag Telos, 1996, ISBN: 3540585419, 978-3540585411

[4] H.G. Paretzke in G.R. Freeman (ed.) Kinetics of inhomogenieous processes, John Wiley \& Sons, New York, 1987, p. 89

[5] W.E. Wilson, H. Nikjoo, Radiat. Envir. Biophys. 38 (1999) 97

[6] M. Krämer, G. Kraft, Radiat. Envir. Biophys. 33 (1994) 91

[7] G. Kraft, Progr. Part. Nucl. Phys. 45 (2000) 473

[8] H.H. Rossi, in: Radiation Dosimetry, F.H. Attix, W.C. Roesch (eds.), Academic Press New York, 1968, vol. I p. 43

[9] H. Rothard, B. Gervais, at. Fys. Medd. Dan. Vid. Selsk. 52 (2006) 497

[10] D.A. Dahl, Int. J. Mass Spectrom. 200 (2000) 3

[11] M. Krämer, Nucl. Instrum. and Meth. B 105 (1995) 14

[12] N. Stolterfoth, R.D. DuBois, R.D. Rivarola, Electron Emission in Heavy lon-Atom Collisions, Springer Series on Atoms and Plasmas Vol. 20, Springer Verlag, Berlin, 1997, p.18ff

[13] K. Siegbahn, $\alpha, \beta$, $\gamma$-Ray Spectroscopy, North Holland, Amsterdam, 1979, vol. I

[14] G. P. Williams, http://xdb.lbl.gov/Section1/Sec 1-1.html last retrieved on Nov. 28, 2007 
[15] J. P. Rozet, C. Stephan, D. Vernhet, Nucl. Instrum. and Meth. B 107 (1996) 67 [16] N. Lineva, Dissertation, Technische Universität Darmstadt, 2008, GSI-Report GSI Diss 2008-1 http://www.gsi.de/documents/DOC-2008-Aug-25-1.pdf last retrieved on Aug. 21, 2008 


\section{Figure Captions}

FIG. 1: Electrostatic toroidal electron spectrometer: Electrons emitted from the target (bottom) under all polar angles are energy analyzed and focused onto a channel plate detector with position-sensitive delay-line read-out (not shown in this figure).

FIG. 2 Electron emission yields for $1 \mathrm{keV}$ electron beam impinging on carbon targets as a function of the emission angle. TRAX Monte Carlo simulations (small symbols) are compared to the measured values (large symbols).

Fig3: Left: Measured and calculated energy spectra of electrons emitted in collision of $\mathrm{C}$-ions with $\mathrm{C}, \mathrm{Ni}, \mathrm{Ag}$, and $\mathrm{Au}$-targets. Right: measured and simulated spectra for the $\mathrm{C}+\mathrm{C}$ collision system. Bombarding energies: left: $3.6 \mathrm{AMeV}$, right $11.4 \mathrm{AMeV}$. The structures in the measured spectra are $\mathrm{C}$ and $\mathrm{O}$ Auger lines from adsorbents. 


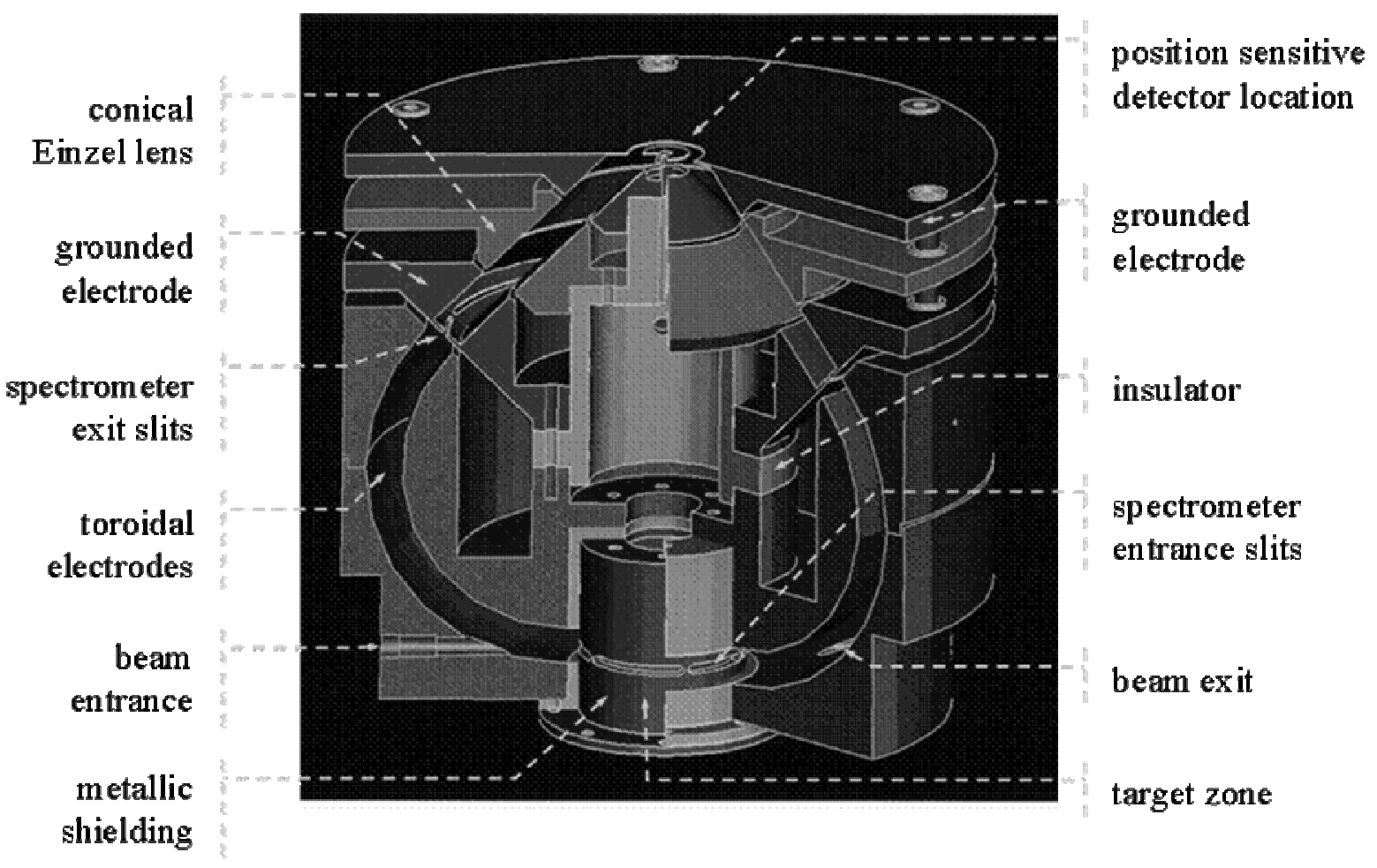

Fig.1

Lineva et al. 


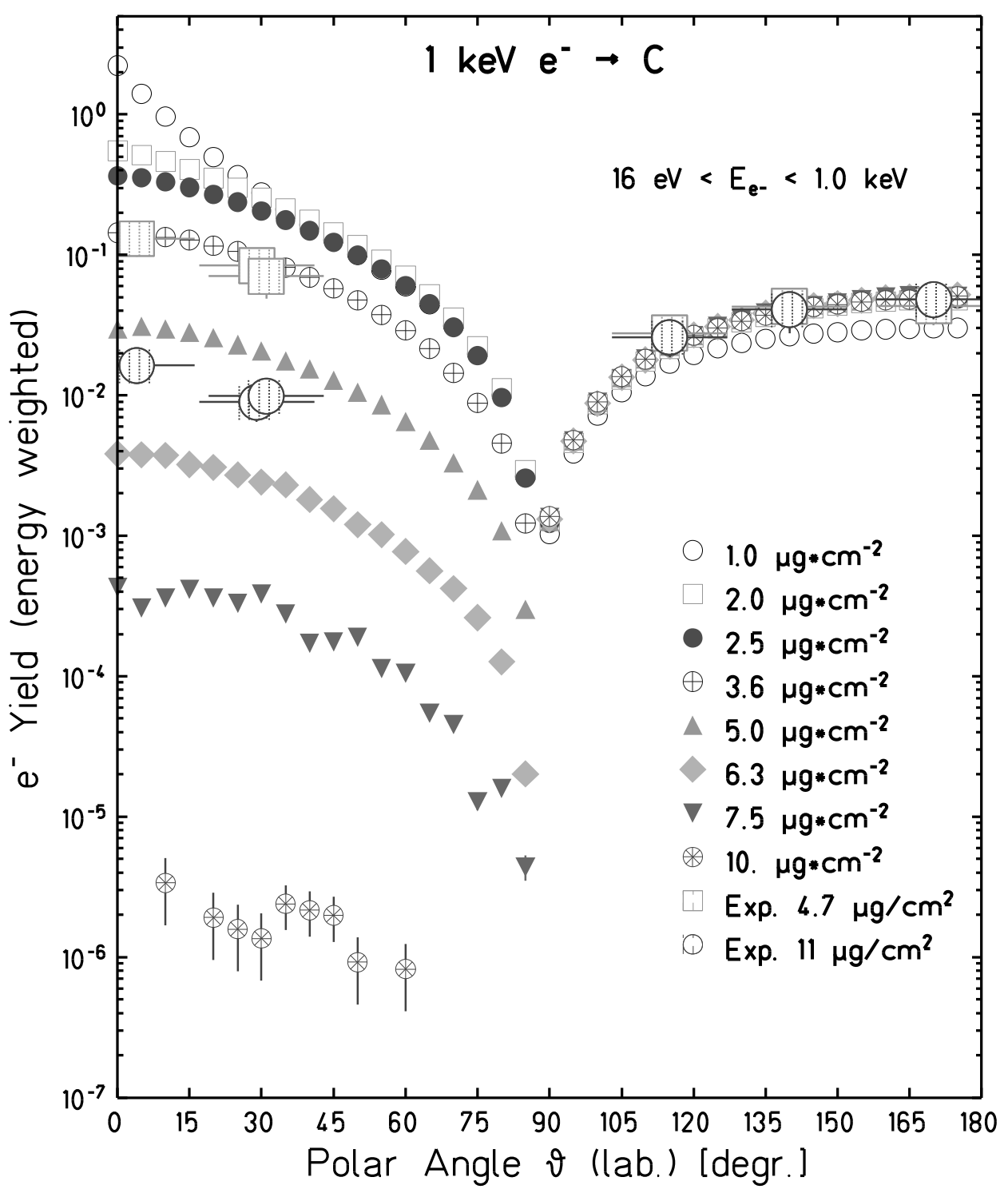

Fig. 2

Lineva et al. 

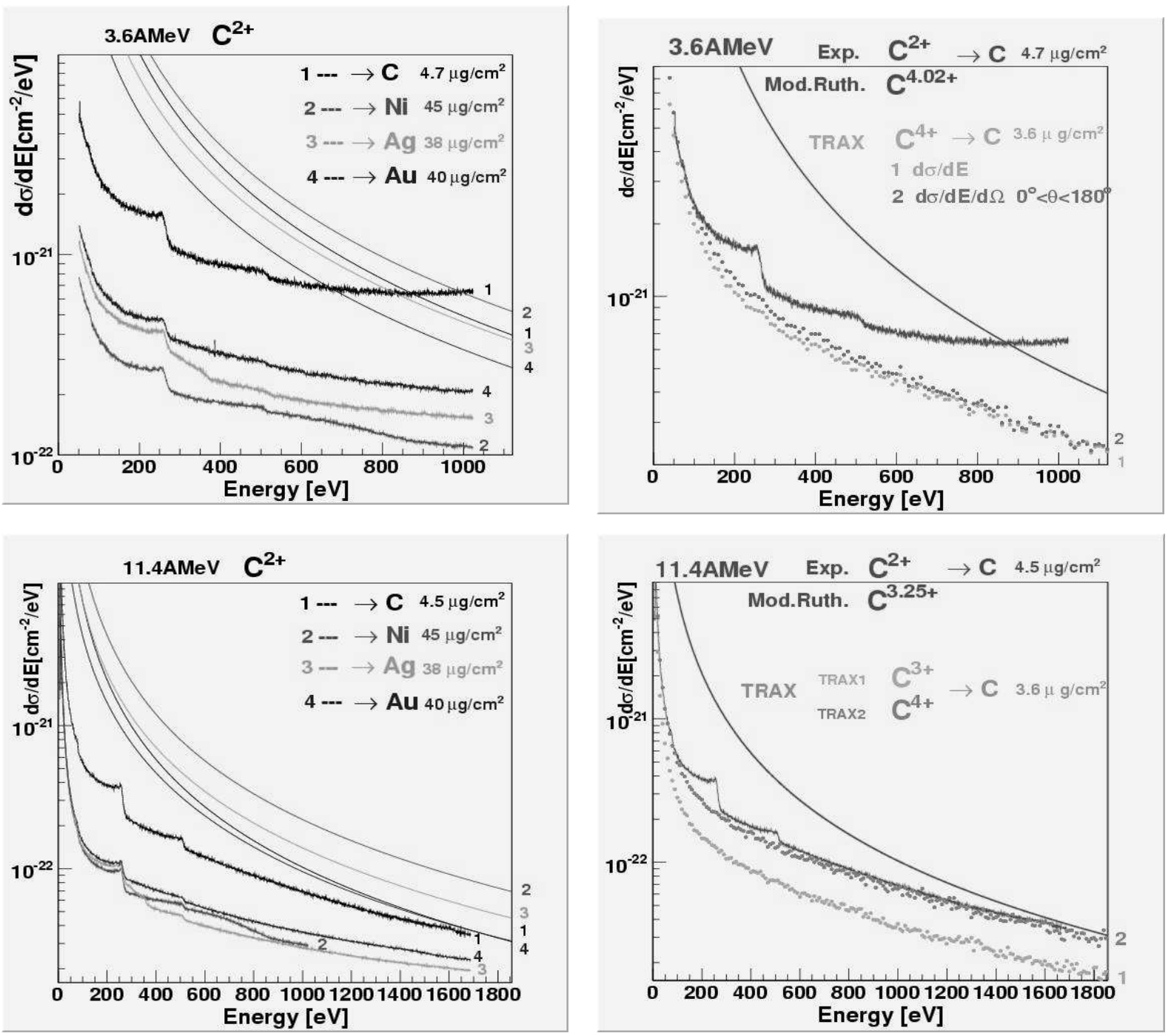

Fig. 3

N. Lineva et al 